\title{
SPECIAL ISSUE: New Insights into Meaning Construction and Knowledge Representation
}

\section{Carolina Rodríguez-Juárez ${ }^{1}$}

\author{
Universidad de Las Palmas de Gran Canaria
}

The ten papers that have been selected for publication in this special issue entitled "New Insights into Meaning Construction and Knowledge Representation" present the outcomes of recent relevant investigations conducted both within Spain and international contexts, and which have been supported by research projects related to various aspects of meaning and knowledge representation. In particular, the findings presented in this volume combine insights from theoretical and computational linguistics in the context of natural language understanding, with parallel studies conducted within the realm of cognitive linguistics with special reference to the role of metaphor and other cognitive operations in meaning construction. Many of the contributions that are presented here are examples of the integration and collaboration between linguistics and other diverse fields such as Natural Language Processing (NLP), semantic memory loss disorders, aeronautic engineering or computer science, that reveal the need to link contemporary linguistics to other arenas that may have a direct and significant impact on society.

The first six papers in this special issue deal with investigations that have been carried out within the framework of the multipurpose lexico-conceptual knowledge base for NLP, FunGramKB, which has mainly been developed over recent years by Periñán-Pascual (Periñán-Pascual \& Arcas-Túnez, 2004, 2007, 2010, 2014; Mairal Usón \& Periñán-Pascual, 2009; Periñán-Pascual \& Mairal Usón, 2009; PeriñánPascual, 2012, 2013) and that interested readers can visit on www.fungramkb.com where they can learn about its modular structure and explore the roadmap to FunGramKB presented by Periñán-Pascual. The first two papers in this group deal directly with the latest research projects that have been carried out within the framework of ARTEMIS (Automatically Representing Text Meaning via an Interlinguabased System), a parsing device that incorporates the analytical tools of Role and

Corresponding author - Universidad de Las Palmas de Gran Canaria, Departamento de Filología Moderna, Calle Pérez del Toro, 1, 35004, Las Palmas de Gran Canaria (Gran Canaria), Spain.

Email: carolina.rodriguez@ulpgc.es 
Reference Grammar (RRG) (Van Valin \& LaPolla, 1997; Van Valin, 2005) and that FunGramKB exploits in order to automatically transduce fragments of natural language and generate their corresponding semantic representation under the format of a conceptual logical structure.

Firstly, in their contribution, "ARTEMIS: State of the art and future horizons", Ana Díaz Galán \& $\mathbf{M}^{\mathbf{a}}$ Carmen Fumero Pérez provide an overview of the latest investigations that have been carried out in the development of the computer application ARTEMIS and focus on the adjustments that need to be incorporated within one of its components, namely the Grammar Development Environment (GDE) where the computational parsing of the morphosyntactic structure of sentences is conducted based on the principles of RRG. Their description is restricted to the parsing of simple sentences in the English language as regards both the theoretical construct that stores the catalogue of Attribute-Value Matrixes (AVMs) which account for grammatical units and where the grammatical features of different constituents are found, along with the construct that compiles the set of grammatical, lexical and constructional production rules which are necessary for the parsing of natural language expressions. Similarly, $\mathbf{M}^{\mathbf{a}}$ Auxiliadora Martín Diaz' paper, "An account of English YES/NO interrogative sentences within ARTEMIS", contributes to the development of ARTEMIS by conducting a thorough analysis of Yes/No interrogative sentences in RRG terms so that all the essential information that is provided by the RRG operators is encoded in their corresponding AVMs for the different relevant categories. In addition, for the implementation of Yes/No questions in the GDE construct in ARTEMIS, the author has had to design a set of production rules that are necessary for the correct parsing of interrogative sentences. All in all, her research offers a comprehensive computational account of Yes/No interrogative sentences in English.

The next two articles in this volume are also in line with recent investigation conducted under the auspices of FungramKB and deal with research carried out in the field of ontology enhancement. Accordingly, the paper "Automatic domainspecific learning: towards a methodology for ontology enrichment" presents a method for the automatic expansion of ontologies developed by Pedro Ureña Gómez-Moreno \& Eva Mestre-Mestre. In their work, they provide a clear account of the difficulties encountered in the extraction of meaningful patterns from massive data, and support the belief that ontologies, as universal models of knowledge representation, occupy a significant role in the processing of large-scale data. Consequently, they propose the "ontology enrichment method" (OEM) that is designed to be able to serve as a useful tool for expanding ontologies from expert domains of knowledge and that has proved to be valid for "the automatic classification of specialized units into a reference ontology". Ángel Felices-Lago \& 
Ángela Alameda-Hernández, in their paper "The process of building the upperlevel hierarchy for the aircraft structure ontology to be integrated in FunGramKB", contribute to the enhancement of FunGramKB by presenting a new domain-specific ontology, which at this stage of their research is limited to the development of its taxonomic hierarchy. Based on their previous experience in the development of subontologies, and thus trying to overcome the problems encountered in the process, they have opted to build the upper-level hierarchy for the aircraft structure ontology, which is based on the English-based controlled language ASD Simplified Technical English, and which is an example of transferring linguistic knowledge to other disciplines, such as aeronautical engineering, since the aircraft structure ontology is intended to provide support for aircraft maintenance management systems. This paper clearly describes the methodology followed, which exploits two of the NLP tools that FunGramKB offers in order to extract terms: on the one hand, DEXTER, an online multilingual workbench designed for the discovery and extraction of terms, and, on the other, DAMIEN, an online workbench that can be used to carry out corpus-based analytics.

The following paper is directly related to one of these NLP tools within ARTEMIS: DAMIEN. Carlos Periñán-Pascual's paper, "Bridging the gap within text-data analytics: a computer environment for data analysis in linguistic research", is concerned with the needs and goals of linguists when using computer applications in their corpus-based research. Despite the large amount of software available, Periñán-Pascual's analysis has allowed him to corroborate the need to implement a type of software that meets the demands and expectations of both theoretical and applied linguists, and that also integrates tools from complementary fields. As a result, he presents DAMIEN, a user-friendly online workbench which provides linguists with a complete array of tools for the development of linguistic research within the field of text-data analytics, and which incorporates tools from corpus linguistics (data storage, access and display techniques), natural language processing (pre-processing capabilities), statistics (data analysis methods) and text mining (methods of classification and clustering) as well as simultaneously being able to account for the properties of linguistic data (e.g. the use of XML for data exchange and of data storage for relational databases).

The final paper in this initial group of six studies conducted within the framework of FunGramKB illustrates a practical example of how the core ontology of the knowledge base can contribute to the design of specialised tests for the detection of semantic memory loss in patients with Alzheimer's disease. In her paper, "A contribution of Natural Language Processing to the study of semantic memory loss in patients with Alzheimer's disease", Beatriz Pérez Cabello de Alba corroborates her initial hypothesis that the knowledge base FunGramKB can be 
used in order to carry out studies in semantic memory loss by improving the protocol of semantic features used in the test of semantic attributes for the detection of semantic memory impairment developed by Grasso, Díaz \& Perarita (2011). She claims that the conceptual entries in the core ontology can be used in order to enrich and complete this detection test by using the inheritance and inference relations that are established among the concepts in the ontology and which will allow to determine the point at which the conceptual string may be damaged and whether the loss of an attribute may affect other concepts that include the same attribute.

The seventh paper in this monograph, "A lexical database for public textual cyberbullying detection", deals with a current phenomenon that has arisen as a result of the use of social networks as vehicles for potential cases of cyberbullying. The authors, Aurelia Power, Anthony Keane, Brian Nolan \& Brian O'Neill, are, in the first place, concerned with providing a thorough definition that is linguistically motivated and that delimits the boundaries of what may constitute public textual cyberbullying. They subsequently present a monolingual lexical database in English that can be used in cyberbullying detection, and describe its overall semantic architecture in which both a lemma-based approach and a syntactic approach based on open and close categories are used, and in which the entries are organised into networks of synonyms. This investigation will undoubtedly contribute to helping with procedures of cyberbullying detection and is one more instance of the transference of linguistic knowledge to society.

The last three papers in this special issue present new insights in the field of cognitive linguistics. As we can read in the title "The role of the cognitive model profile in knowledge representation and meaning construction: the case of the lexical item Europe", Przemyslaw Wilk aims at documenting the semantic potential of the lexical item Europe by analysing a collection of news articles taken from The Guardian newspaper in terms of the Theory of Lexical Concepts and Cognitive Models (Evans 2006, 2009). This theory provides the author with the necessary constructs in order to be able to account for the processes of meaning construction and knowledge representation. Thus, based on the theoretical construct of the cognitive model profile, Wilk has constructed two partial cognitive model profiles for the two lexical concepts of the lexical item Europe, and has also been able to account for meaning construction by showing the role that linguistic context (particularly, the co-text) plays in the activation of specific cognitive models in the cognitive model profile of a given lexical unit.

Nikolay N. Boldyrev, Olga G. Dubrovskaya \& Irina Tolmacheva present the results of their investigation which clearly contributes to the field of Sociocultural Cognitive Semantics by addressing the problem of meaning construction and 
language use. Their paper, "Meaning in the mind within the sociocultural commitment of Cognitive Linguistics", focuses on the belief that the generation of the meaning that is located in the mind of speakers in the process of language use is directly related to the individual knowledge that has been acquired by each speaker as representatives of a particular society and culture, thus highlighting the sociocultural perspective as a commitment that needs to be integrated in any fundamental theoretical framework together with the cognitive perspective. In their research, they address the conception of meaning as a dynamic experience construction process with the aim of establishing what language processes are involved in language use and determining "the integrated system of conceptual structuring in discourse".

The last paper in this special issue, "Metaphor as a device for understanding cognitive concepts" presented by S. V. Kiseleva \& N. A. Trofimova, provides a new cognitive approach to the process of metaphorization that intends to replace the classical study of metaphors by the study of "thinking, learning and conceptual modelling of the metaphorization process." In line with this perspective, the authors depict its baseline algorithm, identify complex metaphors and determine the cognitive-semantic characteristics of their functioning. They also present the analysis of metaphorization in the military field based on the cognitive model outlined in their paper.

I would like to finish by thanking, in the first place, the Revista de Lenguas para Fines Especificos for having served as a platform from which we have been able to publish this collection of papers and, secondly, the researchers who have enlightened us with their diversified and enriching linguistic discussions which will hopefully open up new areas of research and will have direct implications in the study of meaning construction and knowledge representation.

\section{References}

Evans, V. (2006). Lexical concepts, cognitive models and meaning construction. Cognitive Linguistics, 17, 491-534.

Evans, V. (2009). How words mean: lexical concepts, cognitive models, and meaning construction. Oxford: Oxford University Press.

Grasso, L., Díaz, M.C. \& Peraita, H. (2011). Deterioro de la memoria semántico-conceptual en pacientes con enfermedad de Alzheimer. Análisis cualitativo y cuantitativo de los rasgos semánticos producidos en una tarea verbal de definición categorial. Psicogeriatría 3(4), 159-165. 
Mairal Usón, R. \& Periñán-Pascual, C. (2009). The anatomy of the Lexicon within the framework of an NLP knowledge base. RESLA 22, 217-244.

Periñán-Pascual, C. (2012). En defensa del procesamiento del lenguaje natural fundamentado en la lingüística teórica. Onomázein 26(2), 13-48.

Periñán-Pascual, C. (2013). Towards a model of constructional meaning for natural language understanding. In B. Nolan \& E. Diedrichsen (Eds.), Linking constructions into Functional Linguistics: The role of constructions in RRG grammars (Studies in Language Series) (pp. 205230). Amsterdam / Philadelphia: John Benjamins.

Periñán-Pascual, C. \& Arcas-Túnez, F. (2004). Meaning postulates in a lexico-conceptual knowledge base. 15th International Workshop on Databases and Expert Systems Applications (pp. 38-42). IEEE, Los Alamitos (California).

Periñán-Pascual, C. \& Arcas-Túnez, F. (2007). Cognitive modules of an NLP knowledge base for language understanding, Procesamiento del Lenguaje Natural, 39, 197-204.

Periñán-Pascual, C. \& Arcas-Túnez, F. (2010). The Architecture of FungramKB. In Proceedings of the 7th International Conference on Language Resources and Evaluation (ELRA), (pp. 26672674). Malta: European Language Resources Association.

Periñán-Pascual, C. \& Arcas-Túnez, F. (2014). The implementation of the CLS constructor in ARTEMIS. In B. Nolan \& C. Periñán-Pascual (Eds.), Language processing and grammars. The role of functionally oriented computational models (pp. 164-196) Amsterdam / Philadelphia: John Benjamins.

Periñán-Pascual, C. \& Mairal Usón, R. (2009). Bringing Role and Reference Grammar to natural language understanding. Procesamiento del Lenguaje Natural 43, 265-273.

Van Valin, R. D. (2005). Exploring the syntax-semantics interface. Cambridge: Cambridge University Press.

Van Valin, R. D. \& LaPolla, R. (1997). Syntax, structure, meaning and function. Cambridge: Cambridge University Press. 